\title{
Analyses of Partial Discharges in Dielectric Samples under DC Excitation
}

\author{
Edward Corr \\ University of Strathclyde, UK \\ edward.corr@strath.ac.uk
}

\author{
W.H. Siew \\ University of Strathclyde, UK \\ wh.siew@strath.ac.uk
}

\begin{abstract}
The main focus of the paper is to develop a better understanding of partial discharges under DC excitation. Partial discharges studied will initially be limited to discharges from welldefined discharge sites. These include corona, surface discharges and internal voids. The samples are first tested under AC excitation as a sense check to ensure the samples yield the expected PD events. The samples were then subjected to DC excitations where the PD events were recorded and subsequently analysed. A number of analysis techniques will be applied to potentially enable the identification and classification of the type of PD event occurring in the DC system under investigation.
\end{abstract}

Index Terms-- HVDC, Partial Discharge, Condition monitoring, Cables.

\section{INTRODUCTION}

HVDC power systems are likely to form a major and important part of the UK's electricity supply infrastructure over the next decade and beyond. The drivers for the development of HVDC systems in the UK are; the increased importance in harnessing power generated by isolated renewable resources (offshore wind generation) and the advent of voltage source convertor (VSC) technology. The investigation of partial discharge (PD) in such systems is an important area of research as the emission of PD is an indicator of insulation failure in the HVDC system. Any downtime for the system brings significant cost implications to the system operator (cost of repair and lost revenue from power transfer activities) and PD from HVDC cables have yet to be fully understood to prevent such downtimes.

PD detection under AC conditions is a well-known and widely used tool for insulation monitoring, with many different technologies being applied according to the requirements of the scenario under investigation. However, even under AC conditions, PD remains a complex phenomenon with many measurement and diagnostic challenges remaining. The onset of insulation degradation under DC conditions is a known area, but requires fundamental understanding of the underlying mechanisms coupled with new approaches to interpret the PD data.

The original body of research into PD under DC conditions has been conducted at the Technical University of Delft a review of this work is summarised in [1]. This paper details the physics and measurement of PD under DC conditions. PD

events

978-1-4673-9682-0/15/\$31.00 (C2015 IEEE were characterised by the magnitude and time of occurrence and some classification techniques were explored.

The majority of work conducted to date has been focused on the analyses and evaluation of PD from controlled samples under AC conditions [2-6]. PD under AC conditions is well understood and is a developed measurement and post analysis process applied to $\mathrm{AC}$ equipment. In contrast under $\mathrm{DC}$ conditions the same measurement tools are used but the post analysis of PD events is understood in less detail, the main method under investigation being the statistical analysis of the PD activity [7-11].

The results presented in this paper include the comparison of known and controlled PD events under AC and DC excitations from corona, surface discharges and internal voids. A key outcome of this paper is the preliminary proposal on how one might distinguish the different discharges from an insulated system when subjected to DC excitation.

\section{AC VS DC PARTIAL DISCHARGE}

To enable the analysis of PD under AC conditions two common methods are used phase resolved PD (PRPD) plots and pulse sequence analysis (PSA). In this paper PRPD plots will be used to confirm the PD behaviour apparent from the samples under test. In order to produce a PRPD plot two key parameters are required; the charge contained in the current pulse emitted from PD producing defect and a phase reference to the $50 \mathrm{~Hz}$ AC sine wave. These parameters are then used to produce PRPD plots for further analysis and identification of different defect types [12]. In contrast under DC conditions PD generally occurs less often and is more likely to occur during voltage changes. The main parameters available for analysis in the DC case are the charge contained in the PD pulse and the time of occurrence. These parameters are more suited to statistical analysis techniques and as such this type of analysis will be adopted within this paper to demonstrate if the different PD sources can be distinguished. Charge histograms for the samples under test were analysed using methods to assess the shape of the distributions. The skewness and kurtosis of the distributions are assessed in this paper. 


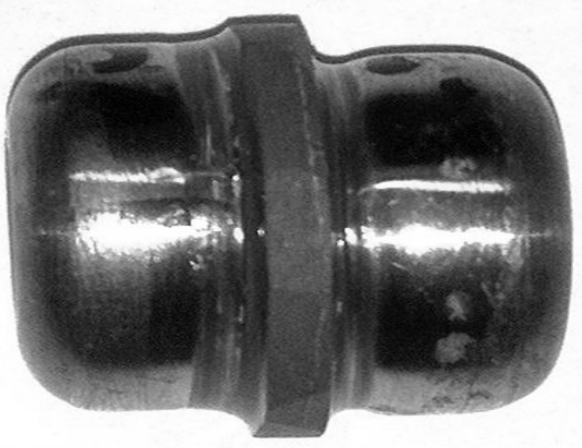

Fig. 1. Single $1.39 \mathrm{~mm}$ void in epoxy with circuit connection allowed via two brass electrodes (19 $\mathrm{mm}$ diameter and $10 \mathrm{~mm}$ thick).

\section{SAMPLES UNDER TEST}

The samples under test in this study are detailed in this section. Three samples were selected to simulate different PD events. The first sample is a $1.39 \mathrm{~mm}$ void in epoxy resin (Fig. 1) to simulate internal discharge, electrical connection was via two plane brass electrodes. The second sample is a ceramic bushing from an $11 \mathrm{kV}$ transformer used to simulate surface discharge. A ground was wrapped around the bushing and additional work was carried out to allow the connection of the supply to the top of the bushing (Fig. 2). The final sample under investigation was that of a positive point plane cell used to initiate corona discharge (Fig. 3). This unconventional point plane arrangement ( $3 \mathrm{~mm}$ point, $40 \mathrm{~mm}$ plane and $10 \mathrm{~mm}$ gap) was used to ensure PD occurred below the maximum output from the DC power supply available.

\section{TEST MEthods}

This section details the test methods adopted when energising the samples under AC and DC stresses.

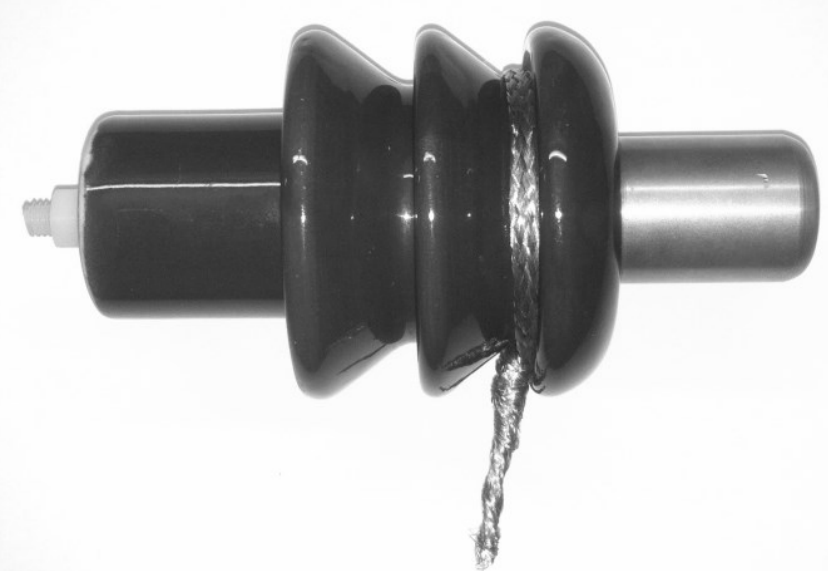

Fig. 2. Surface discharge sample. A ceramic transformer bushing with the high voltage terminal connected via the brass fitting and a ground wrapped part way down the bushing.

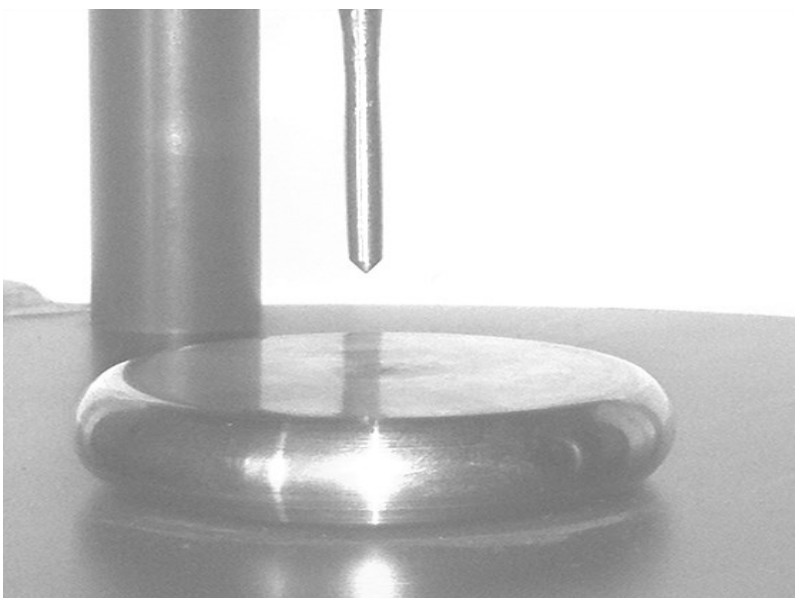

Fig. 3. Point plane in air. $3 \mathrm{~mm}$ point with a 90 degree point and a brass plane electrode (diameter $60 \mathrm{~mm}$ and $10 \mathrm{~mm}$ thick)

\section{A. AC test method}

AC tests were performed using an IEC 60270 standard measurement system (Fig. 4). The measurement system has a bandwidth of 100-400 kHz. The inception voltage was the point at which repetitive and sustained $P D$ was observed on the IEC standard monitoring system. The AC tests were performed by incrementally increasing the supply voltage until repetitive PD was observed on the measurement system. The measurement system was set to record 10 seconds of PD data for the PRPD analysis.

\section{B. DC test method}

The DC tests were performed using the ramp test method adopted from $[13,14]$. This method essentially uses the inception voltage determined under $\mathrm{AC}$ conditions $\left(V_{R}\right.$ was the peak $\mathrm{AC}$ value) as a starting point for performing longer term DC testing on samples. The ramp test profile adopted in this study is detailed in Fig 5 a. The ramp profile consists of periods when the voltage is steadily increased within 30 seconds and periods of 600 seconds where the voltage is held at the required values $\left(V_{R} / 2, V_{R}\right.$ and $\left.3 V_{R} / 2\right)$. The DC tests were performed using an IEC 60270 standard circuit (Fig. 5 b) consisting of a input resistance $\left(R_{i n}\right)$ to decouple the DC power supply from the test circuit, the sample under test $C_{a}$ and a coupling capacitor $C_{k}$. A high frequency current transformer (HFCT) with a transfer impedance of $4.3 \mathrm{~V} / \mathrm{A}$ and a bandwidth of 100 $\mathrm{kHz}$ to $20 \mathrm{MHz}$ was used as the PD sensor. The measurements

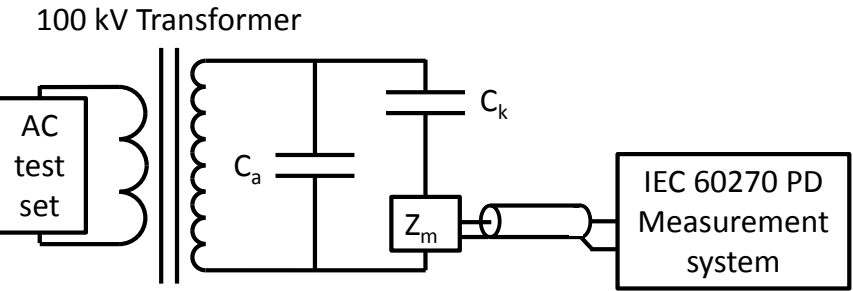

Fig. 4. The AC test circuit with IEC 60270 detection system. The circuit consists of a $C_{a}$ the sample under test, $C_{k}$ the coupling capacitor and $Z_{m}$ the measuring impedance connected to the IEC 60270 PD measurement system. 


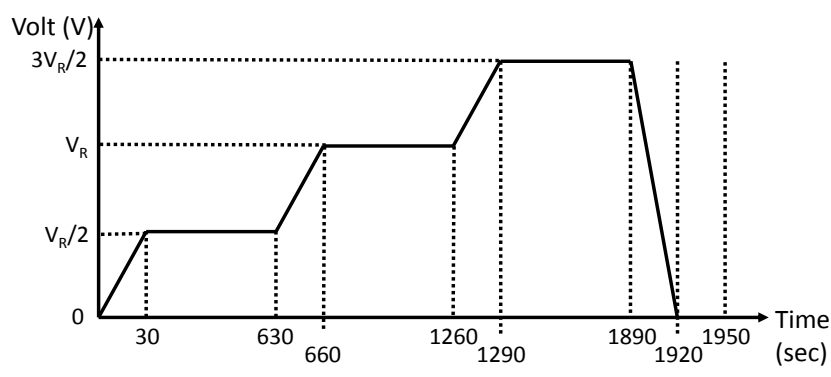

(a)

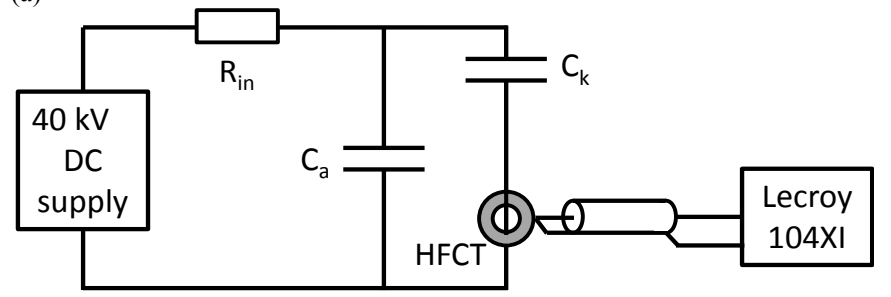

(b)

Fig. 5. (a) The ramp test profile for DC testing. (b) The DC test circuit with HFCT and Lecroy 104Xi oscilloscope for measuring the sensor response. The circuit consists of an input resistance $R_{i n}=25 \mathrm{M} \Omega, \mathrm{C}_{\mathrm{a}}$ the sample under test, $C_{k}$ is the coupling capacitor of $1.9 \mathrm{nF}$ and the $\operatorname{HFCT}\left(Z_{\text {trans }}=4.3 \mathrm{~V} / \mathrm{A}\right)$.

were recorded using a Lecroy $104 \mathrm{Xi}$ oscilloscope with a bandwidth of $500 \mathrm{MHz}$. The oscilloscope was operated in segmentation mode to enable the capture of all positive and negative PD pulses apparent from the sample under test.

\section{RESULTS}

This section details the test results from the $\mathrm{AC}$ and $\mathrm{DC}$ testing of the three controlled samples.

\section{A. Results from AC testing}

The purpose of these tests was to confirm that PRPD plots show that the dominant PD type in the samples was as expected, through reference to available literature [12].

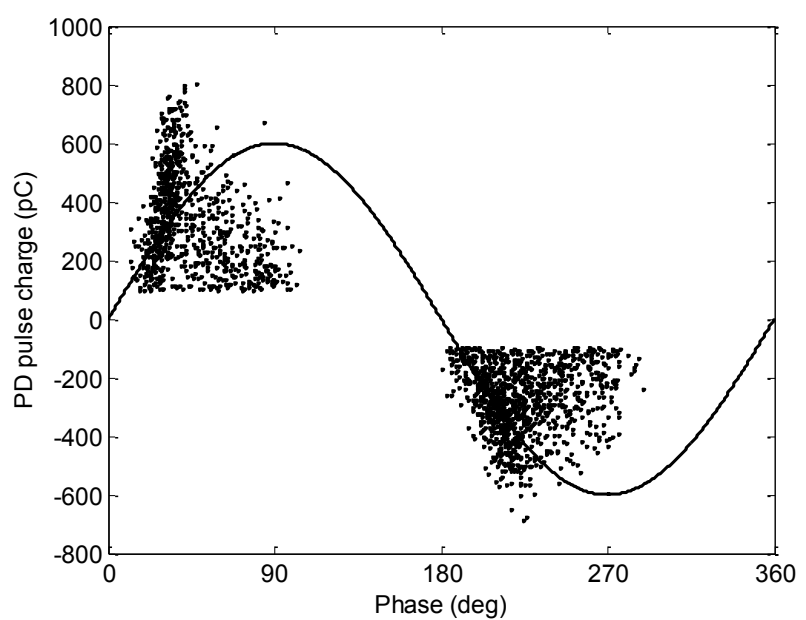

Fig. 6. Single void under AC test at the inception voltage of $7 \mathrm{kV}$. The figure shows that the PD activity is occurring before the test voltage peaks. The peaks are triangular suggesting a large cavity.

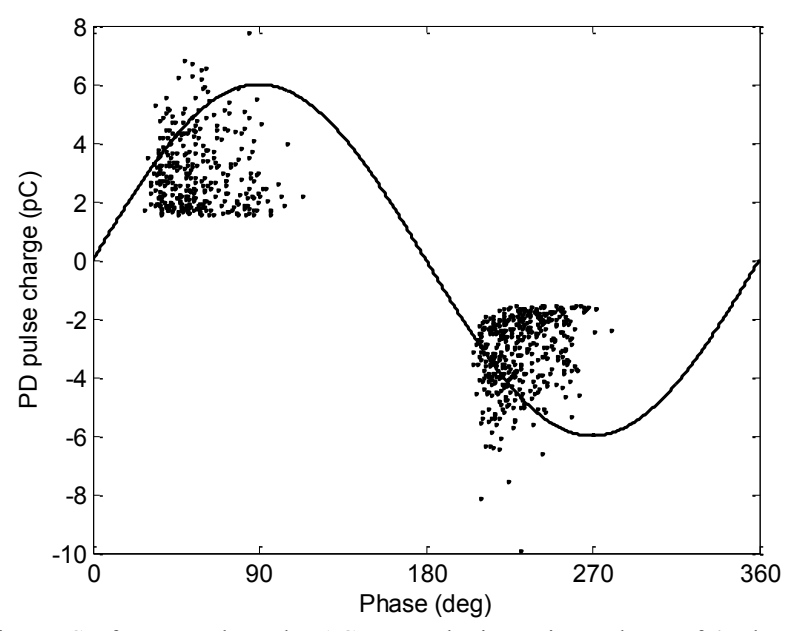

Fig. 7. Surface sample under AC test at the inception voltage of $4.7 \mathrm{kV}$. The figure shows that the PD activity is occurring before the test voltage peaks.

The inception voltage for the single void under $\mathrm{AC}$ conditions was $7 \mathrm{kV}$, the PRPD plot is illustrated Fig. 6. The discharges generally occur in advance of the test voltage peaks, consistent with internal discharge. The peaks are triangular suggesting a large cavity where the discharges can expand.

The inception voltage of the surface sample was $4.7 \mathrm{kV}$. The PRPD plot (Fig. 7) shows that the discharges generally occur before the test voltage peak. The PRPD plot also shows a smaller number of larger discharges in the positive half cycle and a larger number of smaller discharges in the negative half cycle, consistent with expected behaviour for surface discharge.

The inception voltage for the point plane test cell was 5.8 $\mathrm{kV}$. The PRPD plot (Fig. 8) shows a significant peak at 90 degrees matching that of the test voltage peak. The nonstandard point plane sample produced no PD at an increment below $(0.2 \mathrm{kV})$ the determined inception point and at the inception point pulses at $2-4 \mathrm{nC}$ were detected. Generally under $\mathrm{AC}$ conditions small amplitude $\mathrm{PD}$ was expected in the

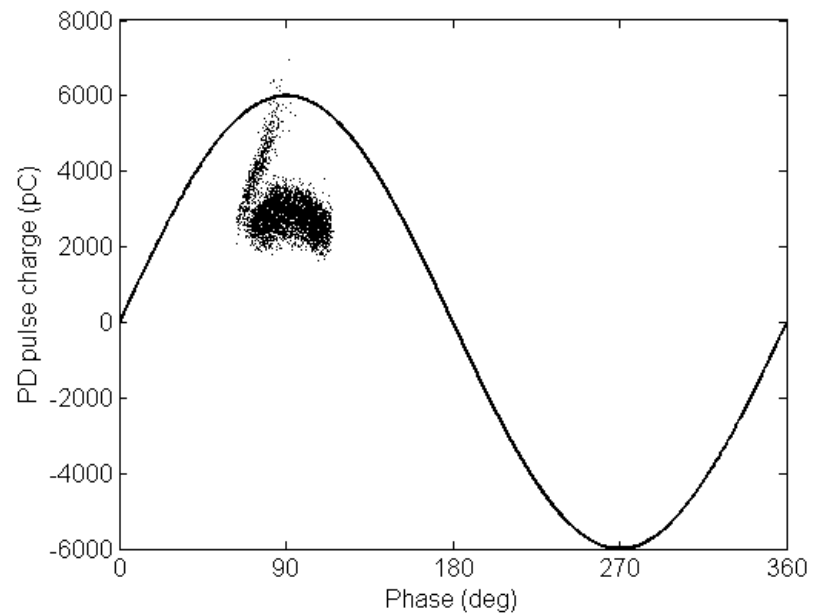

Fig. 8. Point plane under $\mathrm{AC}$ test at the inception voltage of $5.8 \mathrm{kV}$. PD activity is only apparent in the positive half cycle (centered on 90 degrees) it was expected that there was small levels of PD not apparent in the negative half cycle (centered on 270 degrees). 


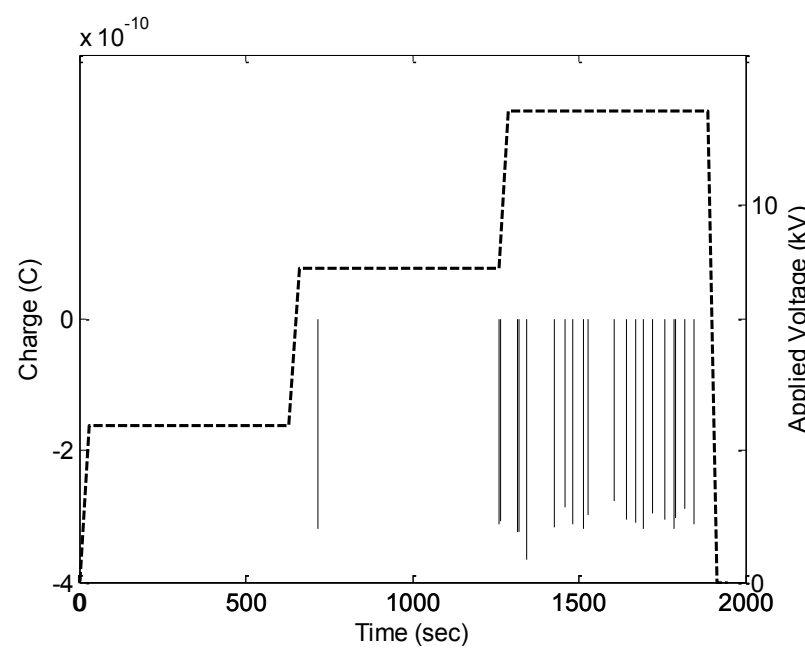

Fig. 9. The ramp profile for the single void. The charge levels from PD activity are shown by the solid lines (y-axis on LHS) and the voltage ramp profile is the dashed line (y-axis on RHS).

negative half cycle this was not apparent due to the large amplitude of PD in the positive half cycle.

The PRPD plots for the three samples tested all behave as expected and match findings in literature.

\section{B. Results from DC testing}

The supply voltage applied to the three samples was varied according to the ramp test profile detailed in Fig. 5a throughout this the PD apparent from the sample under test was recorded.

The ramp profile for the single void sample is illustrated in Fig. 9 detailing the charge of the 21 voltage pulses detected. A single pulse was detected during the hold at $V_{R}(9.9 \mathrm{kV})$ with the majority of activity occurring at the ramp up in voltage and the hold at $3 V_{R} / 2(14.9 \mathrm{kV})$. The histogram of pulse charge is illustrated in Fig 10. The single void sample suffered from significantly reduced PD activity even when the sample was grounded for a week in an attempt to discharge the sample.

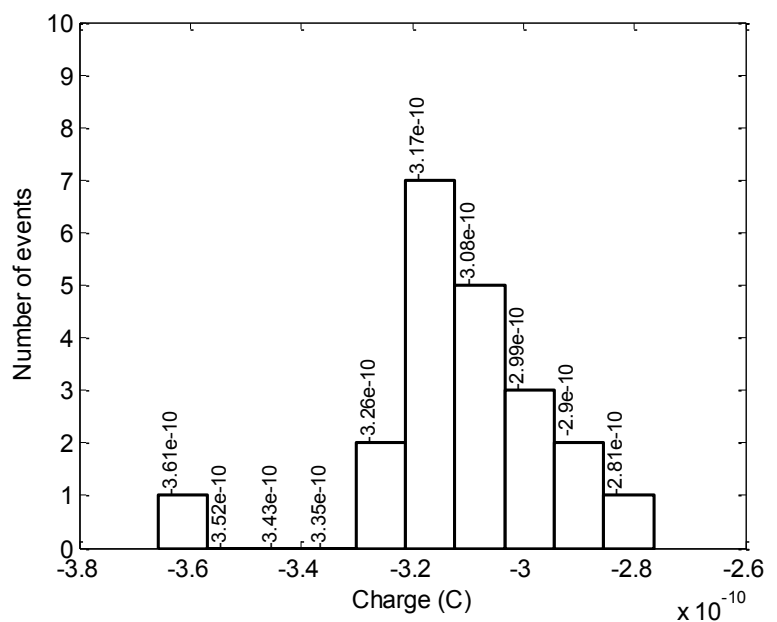

Fig. 10. The pulse charge histograms for the single void sample (21 events). The bin center is detailed above each bar of the histogram.

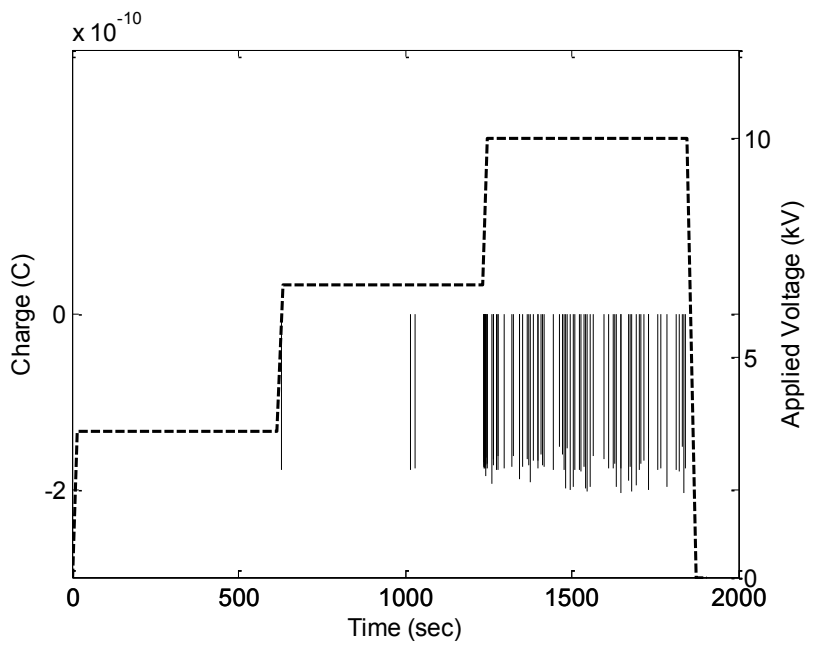

Fig. 11. The ramp profile for the surface sample. The charge levels from PD activity are shown by the solid lines (y-axis on LHS) and the voltage ramp profile is the dashed line (y-axis on RHS).

Investigations are ongoing into aiding charge mobility during the discharge period.

The surface discharge sample ramp profile is shown in Fig. 11 detailing the charge of the 84 voltage pulses detected. The figure shows an initial pulse on the ramp up to $V_{R}(6.7 \mathrm{kV})$ and two subsequent pulses during the hold at $V_{R}$. Regular PD was apparent during the ramp up from $V_{R}$ to $3 V_{R} / 2(10 \mathrm{kV})$ and the hold at $3 V_{R} / 2$. The histogram for pulse charge is illustrated in Fig 12.

The ramp profile for the point plane sample is detailed in Fig. 13 the plot details the charge levels of the 4000 PD events that occurred on the ramp up to $V_{R}(4.2 \mathrm{kV})$ this activity led to saturation of the measurement system in 2 seconds (max allowable samples for post processing). The histogram for the pulse charge during the ramp up to $V_{R}$ is illustrated in Fig. 14. During the ramp up to $V_{R}$ the point plane sample produced regular PD activity and 4000 samples were recorded by the oscilloscope over a 2 second period. The subsequent hold at $V_{R}$ yielded 4000 samples in 3 seconds with the PD pulses having

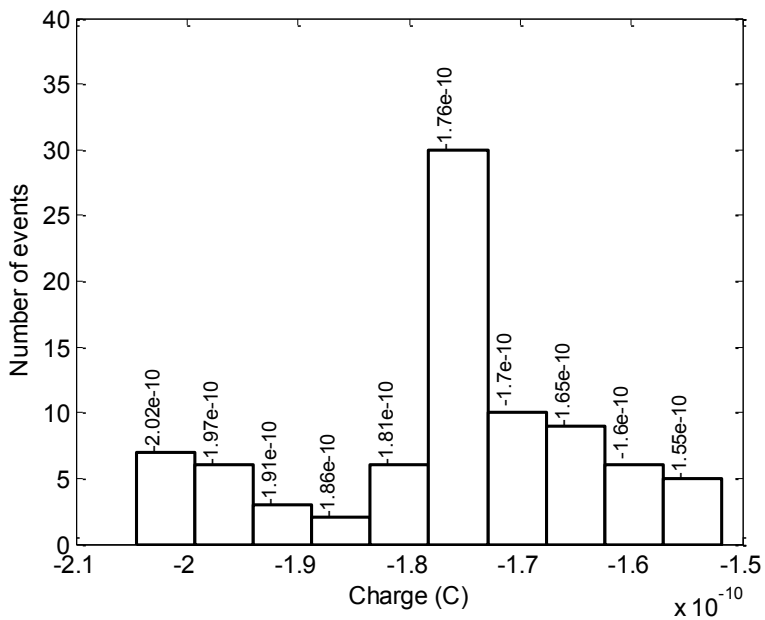

Fig. 12. The pulse charge histograms for the surface discharge sample ( 84 events). The bin center is detailed above each bar of the histogram. 


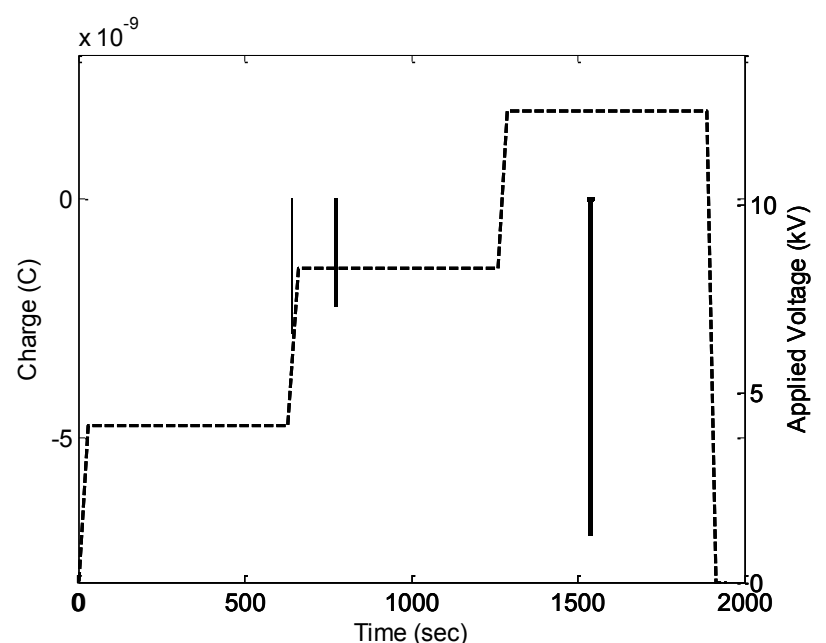

Fig. 13. The ramp profile for the point plane. The charge levels from PD activity are shown by the solid lines detailing the 4000 samples taken at 3 points; the transition to $V_{R}$, hold at $V_{R}$ and hold at $3 V_{R} / 2$ (y-axis on LHS) and the voltage ramp profile is the dashed line (y-axis on RHS).

lower amplitude. At $3 V_{R} / 2(12.5 \mathrm{kV}) 4000$ samples were recorded in 8 seconds with the voltage pulses increasing in amplitude and therefore increased charge levels within these pulses.

The charge histograms were analysed using methods to assess the shape of the distribution namely skewness $(s)$ and kurtosis $(k)$. The skewness (1) is a measure of the symmetry of the distribution around the mean of the sample [15]. A normal distribution (symmetrical distribution) has a skewness of zero using this method.

$$
S=\frac{\frac{1}{n} \sum_{i=1}^{n}\left(x_{i}-\bar{x}\right)^{3}}{\left(\sqrt{\frac{1}{n} \sum_{i=1}^{n}\left(x_{i}-\bar{x}\right)^{2}}\right)^{3}}
$$

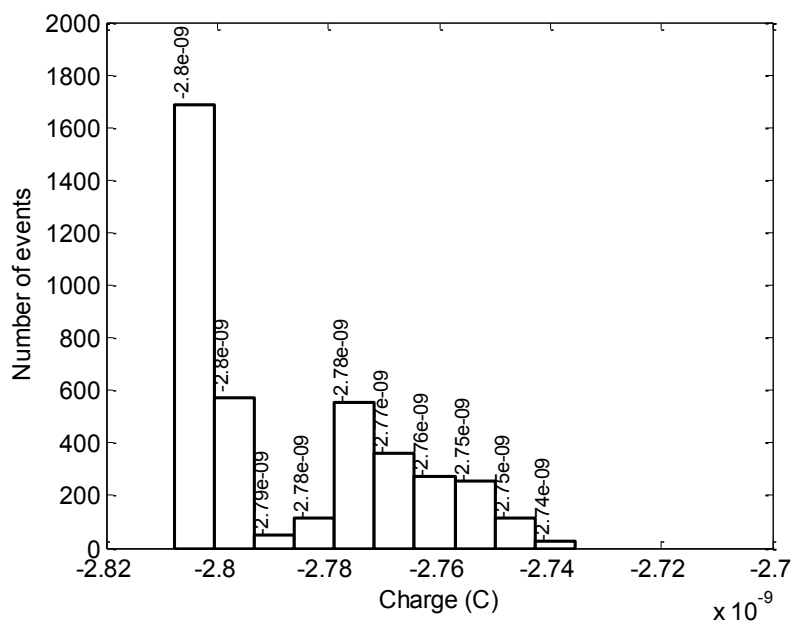

Fig. 14. The pulse charge histograms for the single void sample (4000 events on ramp up to $V_{R}$ ). The bin center is detailed above each bar of the histogram
The kurtosis (2) is a measure of the sharpness of the peak of a distribution, when compared to a normal distribution [15]. In a normal distribution using this method the kurtosis is 3 .

$$
k=\frac{\frac{1}{n} \sum_{i=1}^{n}\left(x_{i}-\bar{x}\right)^{4}}{\left(\frac{1}{n} \sum_{i=1}^{n}\left(x_{i}-\bar{x}\right)^{2}\right)^{2}}
$$

These two methods were applied to assess the distributions of the three charge histograms. Fig.15 details a plot of skewness vs kurtosis for the charge histograms of the three samples under test. The identification of defect types could be possible through using classification techniques with defined boundaries within a skewness vs kurtosis plot. An interesting avenue for further work is the application of the same methods but using a wide band measurement setup to look at the pulse shape characteristics.

\section{DISCUSSION}

The AC testing proved to be a good sense check prior to DC testing to ensure the PD apparent from the samples was consistent with what was expected under AC conditions.

The use of the inception voltage under $\mathrm{AC}$ as an input to the DC ramp test enabled the voltage applied under DC conditions. The ramp test proved to be a controlled method for varying the voltage applied to the sample under test. The Lecroy $104 \mathrm{Xi}$ oscilloscope operating in sequence mode enabled the recording of the PD events apparent from the sample under test. An improvement to ensure that a statistically useful number of PD events are recorded would be to modify the ramp test method so that at $3 V_{R} / 2$ the sample is left energised until 1000 events have been recorded. For all the controlled samples no PD was detected at $V_{R} / 2$ during the ramp testing with PD more likely to occur at $V_{R}$ and $3 V_{R} / 2$.

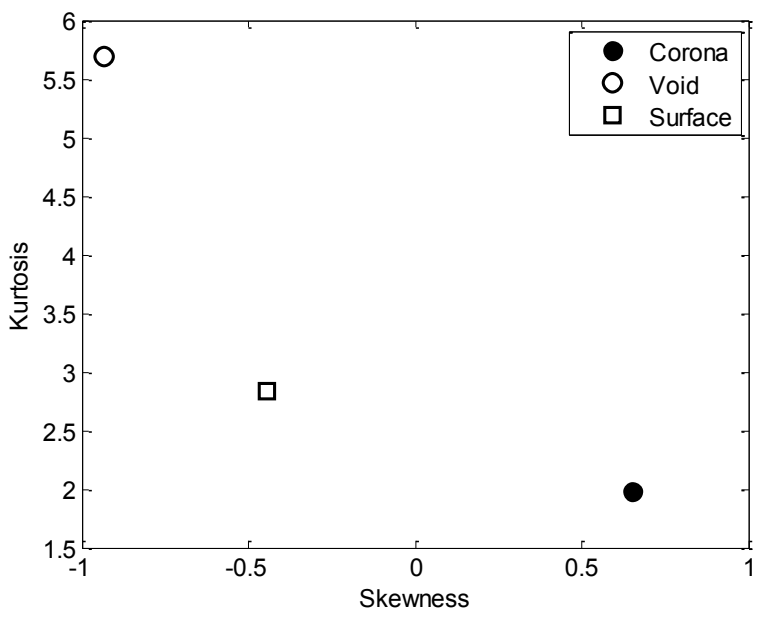

Fig. 15. Skewness versus kurtosis for the charge histograms (corona, void and surface samples). The plot shows that clustering techniques could be developed to enable the defect types to be separated and identified. 
Charge magnitude histograms were constructed using the voltage pulses detected during the voltage ramp testing. Statistical methods (skewness and kurtosis) were applied to the charge histograms. A plot of skewness versus kurtosis illustrated that the three PD sources produce charge histograms of different shapes, which could allow future classification of defect type.

The non-standard point plane sample proved to be very active under AC and DC conditions with high repetition rates and charge magnitudes being recorded. The non-standard sample was used as no PD was apparent from a standard point plane test cell up to the $40 \mathrm{kV}$ maximum supply voltage. The high activity level allowed a large number of samples to be gathered in a short space of time but prevented the full recording of data during the $\mathrm{DC}$ ramp testing.

A key development would be to increase the bandwidth of the measuring circuit. An area of focus is the sensor as the limited bandwidth is not practical for attempting to record accurate PD pulse shapes from the samples under test.

The de-energisation of the samples (in particular the single void) requires further investigation, with the aim of ensuring the sample is in a suitable state to begin/continue further PD testing.

\section{CONCLUSIONS}

This paper has demonstrated the process of first testing controlled samples under AC conditions to ensure the PD behaviour was as expected with verification via the analysis of the PRPD plots. Samples were subsequently tested under DC conditions.

A ramp test method was adopted for the DC testing of controlled samples with the peak value of the $\mathrm{AC}$ inception voltage a key input in determining the voltage steps applied during the ramp test.

The experimental work completed in this paper has highlighted the need to perform longer term tests on samples under DC excitation (tens of minutes) as opposed to the relatively short term test conducted under AC conditions (10 seconds)

Statistical analysis was applied to the charge magnitude of the detected PD pulses from the three controlled samples. The shape of the distributions was analysed using methods to assess the skewness and kurtosis of the distribution.

\section{ACKNOWLEDGEMENTS}

The $\mathrm{PhD}$ research is funded by the EPSRC, project reference number EP/G037728/1 and is done in collaboration with High Voltage Partial Discharge Ltd (HVPD). The authors would like to thank Dr Martin Judd, Xiao Hu and Minan Zhu for their support.

\section{REFERENCES}

[1] P. H. F. Morshuis and J. J. Smit, "Partial discharges at de voltage: their mechanism, detection and analysis", IEEE Transactions on Dielectrics and Electrical Insulation, vol. 12, issue 2, pp. 328-340, April 2005.

[2] D. Adhikari, D. M. Hepburn and B. G. Stewart, "Characterisation of PD Occurring in Injected Air Bubbles in Block Polymeric Insulation”, 2012 Annual Report Conference on Electrical Insulation and Dielectric Phenomena (CEIDP), Montreal, pp. 48-51, October 2012.

[3] A. A. AI-Arainy, N. H. Malik and M. K. AI-Bahloul, "Statistical Variation of ac Corona Pulse Amplitudes in Point-to-plane Air Gaps", vol. 24 , issue: 4, pp. 681-687, August 1989.

[4] H. Illias, T.S. Yuan, A.H.A. Baker, H. Mokhlis, G. Chen and P.L. Lewin, "Partial Discharge Patterns in High Voltage Insulation", 2012 IEEE International Conference on Power and Energy (PECon), Kota Kinabalu, pp. 750-755, December 2012.

[5] S. Kornhuber, M. Boltze, R. Haller, P. Mraz and j. Pihera, "PD Behaviour of Basic Test Arrangements under Different Measurement Conditions", 2012 IEEE Conference on Condition Monitoring and Diagnosis, Bali, pp. 557-560, September 2012.

[6] J.A. Ardila-Rey, J.M. Martinez-Tarifa, G. Robles and M.V. RojasMoreno, "Partial Discharge and Noise Separation by Means of Spectralpower Clustering Techniques", IEEE Transactions on Dielectrics and Electrical Insulation, vol. 20, issue 4, pp.1436-1443, August 2013.

[7] R.J. Van Brunt, "Stochastic properties of partial-discharge phenomena", IEEE Transactions on Electrical Insulation, vol. 26, issue 5, pp. 902948, October 1991.

[8] T. Klueter, J. Wulff and F. Jenau, "Measurement and Statistical Analysis of Partial Discharges at DC Voltage", 2013 48th International Universities' Power Engineering Conference (UPEC), Dublin, pp. 1-5, September 2013.

[9] S. Wenrong, L. Junhao, Y. Peng and L. Yanming, "Digital Detection, Grouping and Classification of Partial Discharge Signals at DC Voltage", IEEE Transactions on Dielectrics and Electrical Insulation, vol 15 , issue 6, pp. 1663-1674, December 2008.

[10] H.Q. Niu, A. Cavallini and G.C. Montanari, "Identification of partial Discharge Phenomina in HVDC Apparatus", IEEE International Symposium on Electrical Insulation, Vancouver, pp. 373-376, June 2008.

[11] P. Morshuis and G. Hoogenraad, "Partial Discharge Diagnostics for DC Equipment", International Symposium on Electrical Insulation, Montreal, pp. 407-410, June 1996.

[12] F.H. Kreuger, "Partial Discharge Detection in High-Voltage Equipment", Butterworth and Co. (Publishers) LTD, London, UK, 1989 , pp. 129-152.

[13] R.S. Bever, J.L. Westrom, "Partial Discharge Testing Under Direct Voltage Conditions", IEEE Transactions on Aerospace and Electronic Systems, Vol. AES-18, Issue1, pp. 82-93, January 1982.

[14] R.S. Bever, "Ramp Technique for Partial Discharge Testing", IEEE Transactions on Electrical Insulation, Vol 20, Issue 1, pp. 38-46, February 1985.

[15] P.R. Hinton, "Statistics Explained", Third ed. Taylor and Francis, Oxford, UK, 2014, pp. 94-100. 